ARTICLE

Received 8 Aug 2013 | Accepted 24 Oct 2013 | Published 15 Nov $2013 \quad$ DOl: 10.1038/ncomms3815

\title{
The N-terminal domains of spider silk proteins assemble ultrafast and protected from charge screening
}

Simone Schwarze ${ }^{1}$, Fabian U. Zwettler ${ }^{1}$, Christopher M. Johnson ${ }^{2} \&$ Hannes Neuweiler ${ }^{1}$

Web spiders assemble spidroin monomers into silk fibres of unrivalled tensile strength at remarkably high spinning speeds of up to $1 \mathrm{~m} \mathrm{~s}^{-1}$. The spidroin $\mathrm{N}$-terminal domain contains a charge-driven, $\mathrm{pH}$-sensitive relay that controls self-association by an elusive mechanism. The underlying kinetics have not yet been reported. Here we engineer a fluorescence switch into the isolated $\mathrm{N}$-terminal domain from spidroin 1 of the major ampullate gland of the nursery web spider E. australis that monitors dimerization. We observe ultrafast association that is surprisingly insensitive to salt, contrasting the classical screening effects in accelerated, charged protein interfaces. To gain deeper mechanistic insight, we mutate each of the protonatable residue side chains and probe their contributions. Two vicinal aspartic acids are critically involved in an unusual process of accelerated protein association that is protected from screening by electrolytes, potentially facilitating the rapid synthesis of silk fibres by web spiders.

\footnotetext{
${ }^{1}$ Department of Biotechnology and Biophysics, Julius-Maximilians-University Würzburg, Am Hubland, 97074 Würzburg, Germany. ${ }^{2}$ Medical Research Council Laboratory of Molecular Biology, Francis Crick Avenue, Cambridge CB2 OQH, UK. Correspondence and requests for materials should be addressed to H.N. (email: hannes.neuweiler@uni-wuerzburg.de).
} 
O rb-web-weaving spiders use seven specialized glands to spin silk threads tailored for various tasks including prey capture, reproduction and shelter ${ }^{1}$. Dragline silk from the major ampullate gland forms the main structural web fibre and spider's lifeline. This thread is exceptionally tough and supersedes mechanical properties of man-made material ${ }^{2-4}$. As such, dragline silk is a focus of biotechnology and biomimetic material sciences. To mimic or reproduce spider silk, it is crucial to understand the structure-function relationship of the constituent proteins, the way they are processed in the spider's spinneret and the interactions they form in fibres. Spider silk fibres are polymers made of protein monomers, so-called spidroins. The bulk of a spidroin sequence consists of repetitive peptide motifs of simple amino-acid composition. This central segment is flanked by non-repetitive, globular carboxy- and amino-terminal domains ${ }^{3,5}$. Spidroins have a molecular weight of about $300 \mathrm{kDa}^{1}$ and the non-repetitive terminal domains cover only $\sim 10 \%$ of the sequence area but have critical roles during silk synthesis ${ }^{5}$. At the beginning of the spinning process, a highly concentrated solution of spidroins is stored in the ampulla of the spinning gland. During silk extrusion, spidroins pass through a tapering duct where they undergo controlled phase and structural transitions before they leave the exit spigot as solid fibre ${ }^{1}$. On their journey through the duct, proteins experience a series of mechanical and chemical stimuli. Shear forces align spidroins mechanically and changes of solution composition induce structural transitions ${ }^{1,4}$. The molecular basis of these transitions is only partly understood. The central sequence segment is largely disordered in the gland but forms regular and non-regular secondary structure in solid silk fibres ${ }^{1,3}$. NMR structural studies show that the carboxy-terminal domain forms a covalent, parallel homodimer that is stabilized by cross-domain disulphides, thus connecting two spidroins covalently ${ }^{6}$. The covalent linkage leads to dimeric strings of spidroins in solution that are terminated by the amino-terminal domains (NTDs) on opposite ends. The NTDs contain a $\mathrm{pH}$-sensitive relay $^{7}$ that triggers anti-parallel homodimerization on solution acidification at physiological $\mathrm{pH}$. A change of $\mathrm{pH}$ from 7 to 6 is induced by proton pumps located at the epithelial cells in the distal part of the spinning duct, just before spidroins leave the exit spigot as silk fibre ${ }^{1,8} \cdot \mathrm{pH}$-triggered NTD dimerization leads to polymerization of spidroins during their passage through the acidified assembly zone, forming supermolecules in solid silk threads ${ }^{7,9,10}$ (Fig. 1). The molecular details of this process are not yet established.

Here we study the kinetics of association of the isolated NTD from spidroin 1 of the major ampullate gland of the nursery web spider Euprosthenops australis by means of fluorescence experiments. We engineer an extrinsic fluorescence switch that signals assembly into the NTD and emulate a change of solution $\mathrm{pH}$, similar to as it happens in the spinning duct, through rapidmixing experiments. We measure association and dissociation rate constants and their dependence on solution ionic strength (IS). We further mutate all protonatable residue side chains of the NTD to probe their contributions to assembly. Two vicinal aspartic acids emerge as critical in a $\mathrm{pH}$-triggered process of accelerated protein association that is surprisingly insensitive to IS. We discuss potential implications of our findings in the rapid synthesis of silk fibres by web spiders.

\section{Results}

Design of a fluorescence reporter for NTD assembly. The NTD of spidroin 1 from the major ampullate gland of the nursery web spider E. australis is a $14-\mathrm{kDa}$ five-helix bundle that forms a homodimer ${ }^{7}$. The anti-parallel symmetry of the association interface brings the side chain of glutamine at position 50

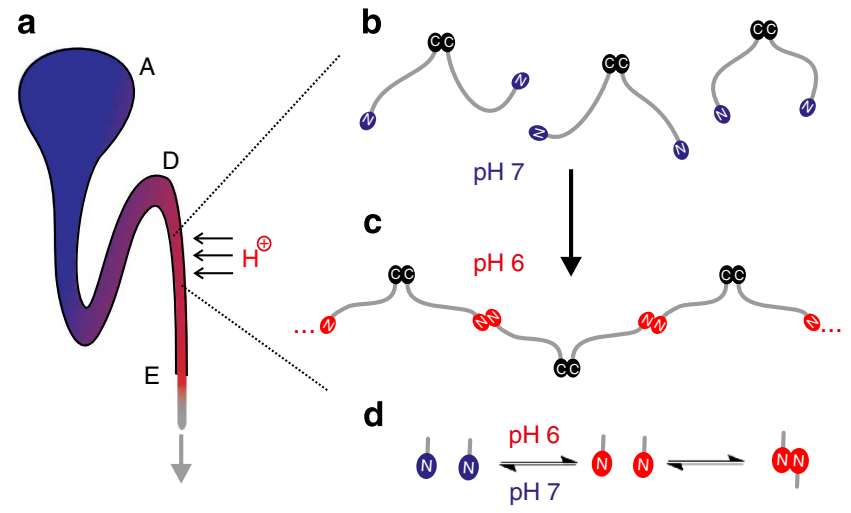

Figure 1 | NTD-assisted association of spidroins in web spiders. (a) Spidroins are stored in the ampulla (A) of the spinning gland. On demand, they pass through a tapering duct (D). Besides various mechanical and chemical stimuli, they experience a change of solution $\mathrm{pH}$ from 7 to 6 (red) in the distal part of the duct and finally leave the exit spigot (E) as solid silk fibre (grey arrow). (b) Spidroins consist of a large central, repetitive sequence motif (grey) that is flanked by small $\mathrm{C}$ - and $\mathrm{N}$-terminal domains (displayed dimensions of the $\mathrm{C}$ and $\mathrm{N}$ domains are not to scale with the repetitive domain). The $\mathrm{C}$ domains form permanent, parallel dimers. (c) The $\mathrm{N}$ domains associate in anti-parallel orientation triggered by solution acidification, which leads to spidroin polymerization. (d) The two-step process of the $\mathrm{pH}$-triggered monomer/dimer equilibrium of the $\mathrm{N}$ domains is illustrated.

(Q50), which flanks the interface, within 1-nm distance to itself on the opposing subunit (Fig. 2a). Organic fluorophores at such short distance form non-covalent dimers with substantially altered absorption and fluorescence emission characteristics ${ }^{11}$. We mutated Q50 to cysteine (Q50C) and chemically modified the mutant at the cysteine side chain with the thiol-reactive, redabsorbing oxazine fluorophore AttoOxa11 (F). Figure 2b shows absorption and fluorescence emission spectra of AttoOxa11modified NTD $\left(\mathrm{NTD}^{\mathrm{F}}\right)$ recorded in buffered solutions where the NTD is monomeric and dimeric, that is, in $50 \mathrm{mM}$ phosphate $\mathrm{pH}$ 7.0 and in $20 \mathrm{mM} 2-(\mathrm{N}$-morpholino)ethanesulphonic acid (MES) $\mathrm{pH}$ 6.0, respectively. At $\mathrm{pH} 7.0, \mathrm{NTD}^{\mathrm{F}}$ shows the common absorption/emission characteristics of AttoOxa11. At $\mathrm{pH}$ 6.0, however, the absorptivity at the maximum wavelength of $\sim 660 \mathrm{~nm}$ is substantially reduced with respect to the lowerwavelength absorption band at $\sim 610 \mathrm{~nm}$. These spectral changes are accompanied by a dramatic loss of fluorescence emission (Fig. 2b) characteristic of dimeric fluorophores in $\pi$-stacking $\mathrm{H}$-type interaction geometry ${ }^{11,12}$. The dramatically changed spectral properties of $\mathrm{H}$-type fluorophore dimers is explained by a delocalized electronic excitation, that is, a molecular exciton ${ }^{11}$. We conclude that AttoOxa11 at position Q50C yields a sensitive and specific probe for NTD dimerization.

Kinetics of NTD association and dissociation. Dimerization of wild-type NTD can be followed by intrinsic fluorescence. A single tryptophan (Trp) side chain at the $\mathrm{N}$ terminus of the NTD is buried in the monomer but changes conformation to take a more solvent-exposed position in the dimer ${ }^{13}$. As a consequence, intrinsic Trp fluorescence emission is quenched on NTD dimerization $7,10,13$. We applied stopped-flow fluorescence spectroscopy to measure kinetics of NTD assembly using both intrinsic and extrinsic probes. We measured association kinetics of wildtype NTD by monitoring Trp and of $\mathrm{NTD}^{\mathrm{F}}$ by monitoring AttoOxa11 fluorescence. Samples were prepared in $10 \mathrm{mM}$ MES $\mathrm{pH} 7.0$ with the IS adjusted to $150 \mathrm{mM}$ and rapidly mixed into 
a
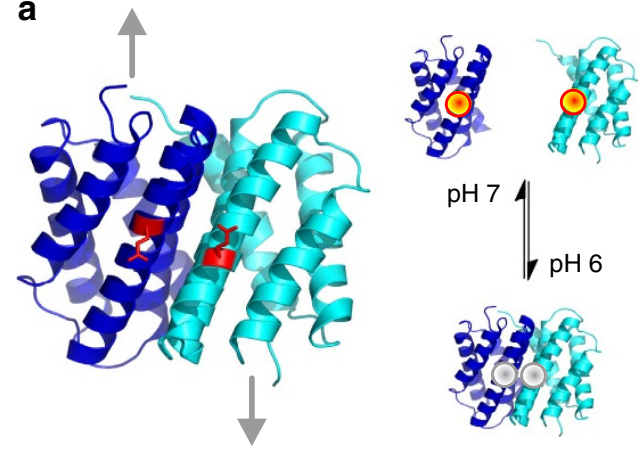

b

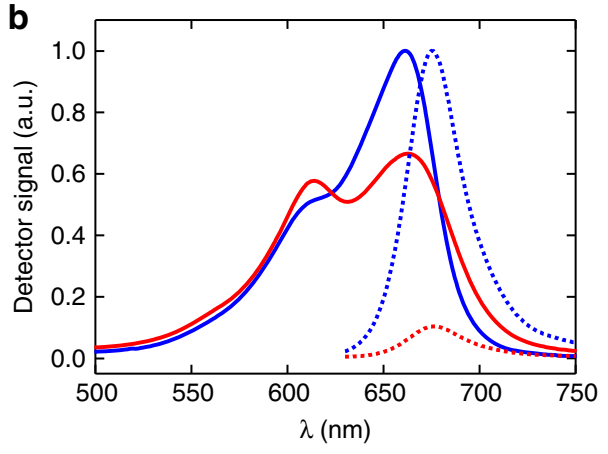

Figure 2 | pH-induced assembly of the NTD monitored by an extrinsic label. (a) Crystal structure of the anti-parallel NTD dimer in cartoon representation with the two subunits coloured blue and cyan (pdb id 3LR2). The positions where central spidroin sequence segments append are indicated by grey arrows. The glutamine side chains of residues Q50 (red) flank the interface and are in close proximity. Chemical modification of the cysteine mutant Q50C with the fluorophore AttoOxa11 (red sphere; $\mathrm{NTD}^{\mathrm{F}}$ ) yields a probe that monitors $\mathrm{pH}$-induced association through formation of $\mathrm{H}$-type fluorophore dimers. (b) Steady-state absorption (solid lines) and fluorescence emission (broken lines) spectra of NTDF measured under monomer ( $\mathrm{pH}$ 7.0, blue) and dimer ( $\mathrm{pH} 6.0$, red) solution conditions.

$50 \mathrm{mM}$ MES pH 6.0 passing through an optical stopped-flow cell. The experiment emulated conditions in the spinneret of web spiders where proton pumps in the distal part of the spinning duct initiate a change of solution $\mathrm{pH}$ from 7 to 6 (refs 1,8 ) (Fig. 1). We observe kinetic transients of fluorescence quenching resulting from NTD association, which fitted well to exponential decay functions (Fig. 3a). Figure $3 \mathrm{~b}, \mathrm{c}$ shows the observed rate constants plotted versus sample concentration. All kinetic transients of association of NTD and NTD ${ }^{\mathrm{F}}$ together with data fits are provided as Supplementary Figs S1 and S2. Observed rate constants increased linearly with sample concentration, as expected for a bimolecular event (Fig. 3b,c). The slopes of linear fits to the data yield association rate constants, $k_{\mathrm{a}}$, of $2.6 \pm 0.5 \times 10^{8} \mathrm{M}^{-1} \mathrm{~s}^{-1}$ for NTD and of $3.0 \pm 0.2 \times$ $10^{8} \mathrm{M}^{-1} \mathrm{~s}^{-1}$ for NTD $\mathrm{NT}^{\mathrm{F}}$. The good agreement of rate constants measured from intrinsic Trp and extrinsic AttoOxal1 fluorescence shows that extrinsic modification does not perturb NTD association.

The NTD dissociation rate constant, $k_{\mathrm{d}}$, cannot be obtained under the physiologically relevant conditions at $\mathrm{pH} 6$ from intrinsic Trp fluorescence. However, the engineered extrinsic probe provides a unique tool to measure $k_{\mathrm{d}}$ at $\mathrm{pH} 6$ using chasing experiments. We prepared dimeric $\mathrm{NTD}^{\mathrm{F}}$ in $\mathrm{pH}$ 6.0-buffered solution and chased one subunit off the dimer by rapidly mixingin excess of non-modified NTD using the stopped-flow technique. Since the monomer/dimer equilibrium is dynamic and since under these conditions $k_{\mathrm{a}}$ is much larger than $k_{\mathrm{d}}$, every dissociation event of dimeric NTD ${ }^{\mathrm{F}}$ results in rapid reassociation with non-modified NTD, present at excess concentration, until the equilibrium is reached and all fluorescence-quenched $\mathrm{NTD}^{\mathrm{F}}$ / NTD $^{\mathrm{F}}$ dimers exchanged to fluorescent $\mathrm{NTD}^{\mathrm{F}} / \mathrm{NTD}$ assemblies. The resulting fluorescence increase signals $\mathrm{NTD}^{\mathrm{F}}$ dissociation. Measured kinetic transients of dissociation fit well to exponential rise functions (Fig. 3d). To further test whether the observed kinetics truly reflected dissociation, we measured transients at various sample concentrations. The $k_{\mathrm{d}}$ determined for 25,50 and $100 \mathrm{nM} \mathrm{NTD}^{\mathrm{F}}$ in $50 \mathrm{mM}$ MES pH 6.0 was $0.3 \pm 0.1,0.33 \pm 0.05$ and $0.26 \pm 0.05 \mathrm{~s}^{-1}$, respectively, values which are within error. Thus, as expected, obtained values of $k_{\mathrm{d}}$ are insensitive to sample concentration.

The influence of IS on kinetics. The monomer/dimer equilibrium of the NTD at pH 6 is dependent on IS ${ }^{9}$. Steady-state fluorescence emission intensities show that at low IS NTD ${ }^{\mathrm{F}}$ is dimeric but at IS $>200 \mathrm{mM} \mathrm{NTD}{ }^{\mathrm{F}}$ is essentially monomeric (Supplementary Fig. S3). We measured $k_{\mathrm{a}}$ and $k_{\mathrm{d}}$ of $\mathrm{NTD}^{\mathrm{F}}$ in pH 6.0-buffered solutions of increasing IS. The results are shown in Fig. 4. Kinetic transients together with fits to the data are provided as Supplementary Figs S4 and S5. $k_{\mathrm{a}}$ is virtually insensitive to salt. By contrast, $k_{\mathrm{d}}$ increases substantially with increasing IS. In fluorescence transients of $\mathrm{NTD}^{\mathrm{F}}$ dissociation at low IS, that is, at IS $<100 \mathrm{mM}$, a second kinetic phase emerges that fits to an exponential rise time, which is two- to fourfold slower than that of the main kinetic phase (Supplementary Fig. S5). This additional phase may originate from the presence of low levels of NTD oligomers. Clustering fluorophores in oligomeric $\mathrm{NTD}^{\mathrm{F}}$ can undergo resonance energy transfer (homo-Förster resonance energy transfer ) leading to quenching of fluorescence and may explain the occurrence of an additional kinetic phase at low IS. In support of this interpretation, higher-order NTD oligomers have previously been observed in light-scattering experiments carried out at $\mathrm{pH} 6$ (ref. 7). However, their presence is controversial as they have not been detected in other experimental studies ${ }^{10,13}$.

From the rate constants $k_{\mathrm{a}}$ and $k_{\mathrm{d}}$ we calculated the equilibrium dissociation constant, $K_{\mathrm{d}}$, which is found to be in the nanomolar range and strongly dependent on IS. $K_{\mathrm{d}}$ provides access to the free energy of $\mathrm{NTD}^{\mathrm{F}}$ dimerization, $\Delta G$. All thermodynamic parameters are summarized in Supplementary Table S1. To test whether the observed changes of $k_{\mathrm{d}}$ and $\Delta G$ resulted from charge screening, we applied the Debye-Hückel formalism in the analysis of the data, which describes the activity of ions in solution $^{14}$. Following this law, a change of $\Delta G$ originating from charge screening is proportional to the square root of IS $\left(\mathrm{IS}^{0.5}\right)^{15,16}$. We plotted $\Delta G$ of NTD assembly versus $\mathrm{IS}^{0.5}$ and found that it changed linearly, in accord with the Debye-Hückel law (Fig. 4, inset). From the linear fit to the data, we extrapolate the dissociation rate constant at zero IS, $k_{\mathrm{d}}^{0}$, to $0.025 \pm 0.006 \mathrm{~s}^{-1}$. The extrapolated free energy at zero IS, $\Delta G^{0}$, is $13.8 \pm 0.2 \mathrm{kcal} \mathrm{mol}^{-1}$.

The influence of NTD side chain charges on kinetics. The 137residue sequence of the NTD contains a total of 12 ionizable amino-acid side chains: eight negatively charged aspartic or glutamic acids (D or E), three positively charged arginine or lysine residues ( $\mathrm{R}$ or $\mathrm{K}$ ) and one histidine $(\mathrm{H})$. To probe their contribution to assembly, we deleted the side chain charges individually on the background of $\mathrm{NTD}^{\mathrm{F}}$ using site-directed mutagenesis. Aspartic and glutamic acids were mutated to the isosteric, non-charged asparagines and glutamines. Histidine, lysine and arginine were mutated to alanine. Kinetic transients of 
a

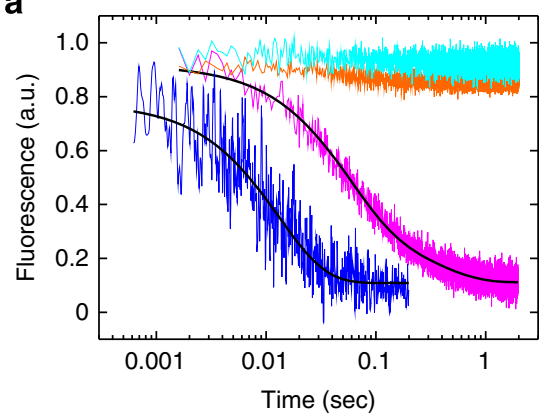

c

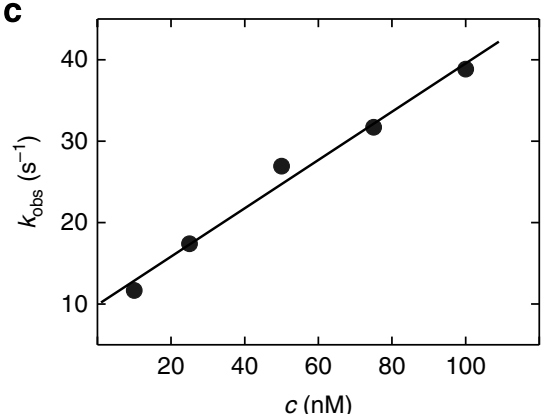

b
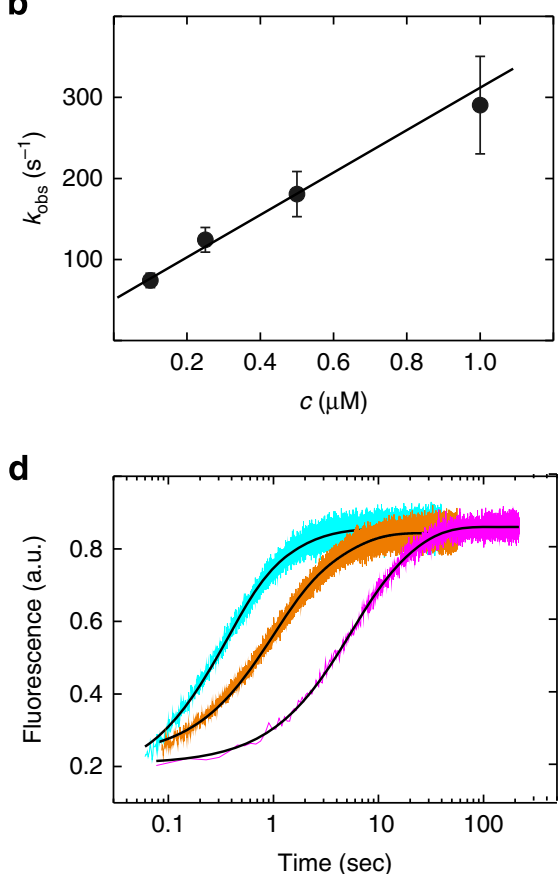

Figure 3 | Association and dissociation kinetics of the NTD. (a) Fluorescence transients of NTD association induced by a rapid change of solution pH from 7.0 to 6.0, measured using stopped-flow spectroscopy. Representative transients of 100 nM NTD monitored by Trp fluorescence (blue, average over seven shots) and $25 \mathrm{nM} \mathrm{NTDF}$ monitored by AttoOxa11 fluorescence (magenta, single shot) are shown. Black lines are data fits to exponential decay functions. In addition, fluorescence transients of NTDF mutants D39N and D40N are shown in orange and cyan, respectively. (b) Observed association rate constants $\left(k_{\text {obs }}\right)$ obtained from Trp fluorescence of NTD and plotted versus sample concentration. The solid line is a linear data fit. Error bars are s.d. of three measurements. (c) Observed association rate constants measured from AttoOxa11 fluorescence of NTDF and plotted versus sample concentration. The solid line is a linear data fit. s.d. of three measurements were on the order of symbol size. (d) Kinetic transients of dissociation of NTDF dimers in 50 mM MES pH 6.0 measured from AttoOxa11 fluorescence using chasing experiments. Representative kinetic transients of NTDF (magenta) and mutants E84Q (orange) and R60A (cyan) are shown. Black lines are fits to exponential rise functions.

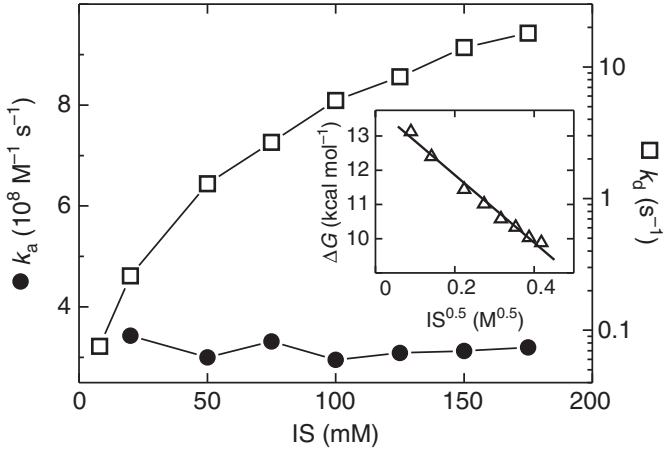

Figure 4 | Influence of IS on kinetics. Association rate constants $k_{a}$ (closed circles) and dissociation rate constants $k_{d}$ (open squares) of NTDF measured at $\mathrm{pH} 6.0$ and increasing IS. The inset shows the free energy of NTD dissociation calculated from $k_{\mathrm{a}}$ and $k_{\mathrm{d}}$ and plotted versus the square root of IS. The solid line is a linear fit to the data.

association and dissociation of $\mathrm{NTD}^{\mathrm{F}}$ mutants were measured in $50 \mathrm{mM}$ MES pH 6.0 using stopped-flow fluorescence spectroscopy. Representative transients are shown in Fig. 3a,d. Values of $k_{\mathrm{a}}$ and $k_{\mathrm{d}}$ were derived in the same way as described above for the non-mutated pseudo wild-type protein. Kinetic transients of all mutants had significant amplitudes, except of D39N and D40N. D39N and D40N had virtually no fluorescence signal change and, thus, no rate constants of association or dissociation could be obtained. The values of $k_{\mathrm{a}}$ and $k_{\mathrm{d}}$ determined for all other mutants are shown in Fig. 5. All rate constants together with derived thermodynamic data are provided in Supplementary Table S1. $k_{\mathrm{a}}$ was between $1-5 \times 10^{8} \mathrm{M}^{-1} \mathrm{~s}^{-1}$ and fluctuates around the value determined for the pseudo wild-type $\mathrm{NTD}^{\mathrm{F}}$ (Fig. 5a). Values of $k_{\mathrm{d}}$ vary with position and are significantly elevated (up to 13-fold) for all mutants (Fig. 5b). Mutant E119Q is an exception as it shows a fivefold decrease of $k_{\mathrm{d}}$ as compared with the pseudo wild-type protein.

\section{Discussion}

The NTD of spidroins evolved to maintain solubility of silk fibrebuilding blocks in the ampulla of the spinning gland and to assist their transformation into threads on demand ${ }^{5}$. The sequence composition of the NTD is unusual and its fold is unique with no homologue identified so far $^{7}$. Notably, only $9 \%$ of NTD aminoacid side chains are ionizable, which is a small number compared with the average $29 \%$ in proteins ${ }^{17}$. This is in particular remarkable, as charged side chains on protein surfaces usually provide water solubility. The side chain charges of the NTD are very likely to have additional roles in spidroin assembly because a protonation event at physiological $\mathrm{pH}$ triggers NTD selfassociation in the spinning duct ${ }^{7,9,10}$. The mechanistic understanding of this process has two aspects: first, the functionality of the $\mathrm{pH}$-sensitive relay that triggers association and, second, the actual association process itself.

NTDs dimerize promoted by a decrease of solution $\mathrm{pH}$ from 7 to 6 (refs 7,9,10) (Fig. 1). Thus, a protonation event acts on monomeric NTD where it triggers a change of structural and/or physicochemical properties that facilitates subsequent association. The site(s) of protonation in the NTD and the mechanism of the 

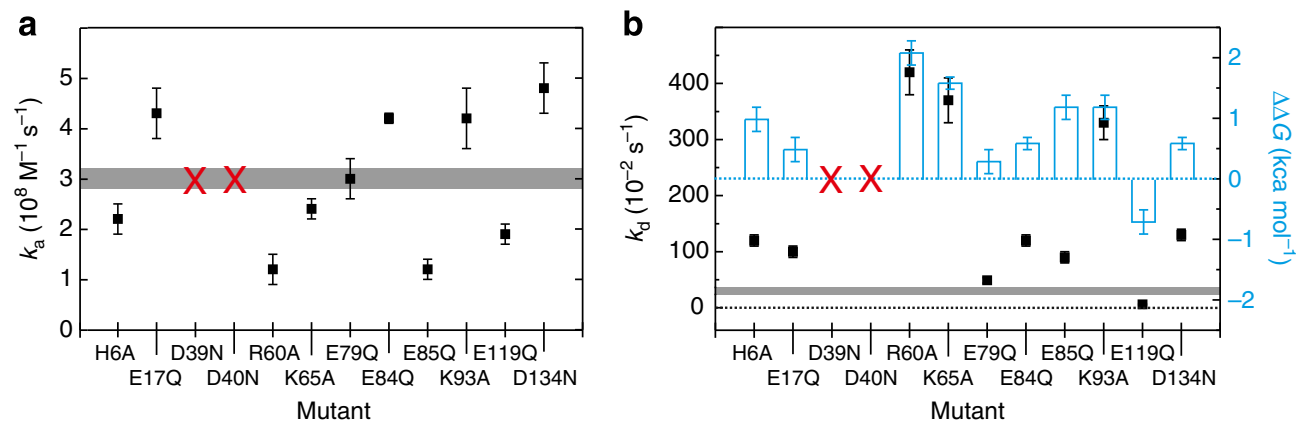

Figure 5 | Influence of side chain charges on kinetics. (a) Rate constants of association of NTDF and mutants thereof determined in 50 mM MES pH 6.0. Error bars are s.e. The grey horizontal line indicates the value of the pseudo wild-type protein NTDF (the width of the line represents the s.e.). (b) Rate constants of dissociation of NTDF and mutants thereof determined in $50 \mathrm{mM} \mathrm{MES} \mathrm{pH} \mathrm{6.0.} \mathrm{Error} \mathrm{bars} \mathrm{are} \mathrm{s.d.} \mathrm{of} \mathrm{three} \mathrm{measurements.} \mathrm{The} \mathrm{grey} \mathrm{and}$ solid horizontal line indicates the value of the pseudo wild-type protein NTDF (the width of the line represents the s.d.). Open cyan bars show the changes of the free energies of dimerization for each mutant calculated from $k_{\mathrm{a}}$ and $k_{\mathrm{d}}$. Quantities for mutants D39N and D40N could not be determined because no fluorescence amplitude was detected (red X). Broken horizontal lines indicate the value zero.
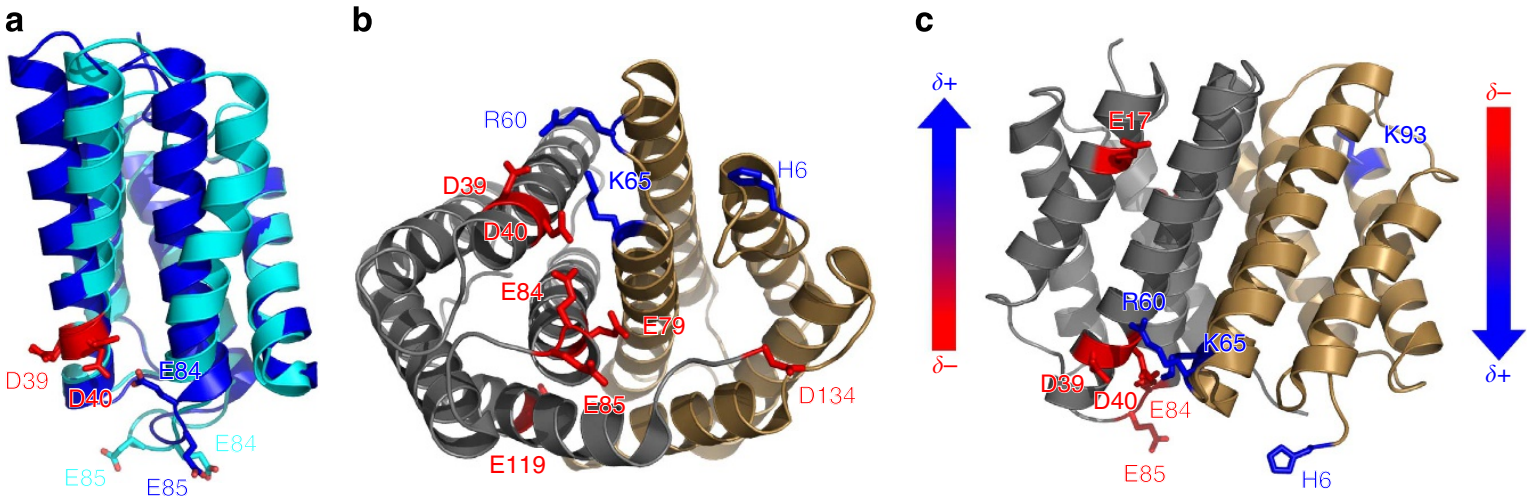

Figure 6 | Distribution of side chain charges in the NTD. (a) Alignment of the solution structure of monomeric NTD at pH 7 (cyan, pdb id 2LPJ) with the crystal structure of dimeric NTD (blue, pdb id 3LR2) reveals distinct conformational changes ${ }^{13}$. Shown is a view onto the association interface. Residues D39 and D40 are highlighted in red. Helices of the interface tilt out in the monomer conformation. The structural element surrounding E84 is a coil in the monomer but a helix in the dimer. (b,c) Location of ionizable amino-acid side chains in dimeric NTD (bottom and side view, pdb id 3LR2). The anti-parallel oriented subunits and coloured grey and brown. Acidic (red) and basic (blue) side chains are depicted in stick representation. The macromolecular dipole moment of each subunit that originates from an uneven distribution of side chain charges, identified in structural studies ${ }^{7}$, is indicated by arrows.

$\mathrm{pH}$ relay are not yet established. To lose or take a proton, the $\mathrm{pK}_{\mathrm{a}} \mathrm{s}$ of involved side chains must lie within the range of the applied $\mathrm{pH}$ change, that is, between 7 and 6 . The normal $\mathrm{pK}_{\mathrm{a}}$ of carboxylic acids in proteins is 3-4 but strongly depends on the local environment ${ }^{17}$. Proximity of negatively charged carboxylates leads to unfavourable Coulomb repulsion and can elevate their $\mathrm{pK}_{\mathrm{a}} \mathrm{s}$ to physiological value, thus alleviating repulsive Coulomb forces through protonation ${ }^{17}$. Askarieh et al..$^{7}$ suggested from the crystal structure of the dimer and mutagenesis experiments that a direct interaction between the D40 and E84 side chain carboxylates could generate a $\mathrm{pH}$ relay. However, a subsequent solution NMR study showed significant structural differences between monomeric and dimeric forms of the $\mathrm{NTD}^{13}$ (Fig. 6a). From the solution structure it is evident that the interaction between D40 and E84, which is present in the dimer, is absent in the monomer. This seems to exclude the D40/E84 interaction as a $\mathrm{pH}$ relay. Here we deleted all 12 side chain charges of the NTD individually and probed their influence on kinetics of dimerization. From our analysis, it emerges that D39N and $\mathrm{D} 40 \mathrm{~N}$ are the only mutants that abolish dimerization (Fig. 5). Both mutants had virtually no fluorescence signal change in rapid-mixing experiments (Fig. 3a). Mutation E84Q, on the other hand, had only a minor effect. The observed inability of D40N to form dimers is in agreement with results from Askarieh et al. ${ }^{7}$ as well as fluorescence and mass spectrometry studies ${ }^{7,18}$. However, the effect of mutating D39 was not examined in these previous studies. Results suggests that D39 and D40 may form a pH relay. The side chains of D39 and D40 are fixed on a helix in close spatial proximity, both in monomeric and dimeric NTD (Fig. 6a). D39 and D40 may be close enough to elevate their $\mathrm{pK}_{\mathrm{a}} \mathrm{s}$ to physiological value. A mutual $\mathrm{pK}_{\mathrm{a}}$-elevating effect would be abolished on mutation of either one of the two side chains to asparagine and protonation-induced dimerization at $\mathrm{pH} 6$ would be abrogated, as observed in our experiments. However, there is an additional side chain that may have a role. E119Q was the only mutation that significantly stabilizes the dimer. The dissociation rate constant of E119Q is fivefold slower than that of the pseudo wild-type protein and provides a stabilizing free energy of $0.7 \pm 0.2 \mathrm{kcal} \mathrm{mol}^{-1}$ (Fig. 5b). It is conceivable that an elevated side chain $\mathrm{pK}_{\mathrm{a}}$ of E119 leads to protonation at $\mathrm{pH}$ 6, thereby removing the charge of the carboxylate. In support of this interpretation, computer simulations predicted a destabilizing penetration of water molecules into the dimerization interface mediated by the E119 carboxylate ${ }^{19}$. However, experimental 
evidence for the protonation of specific side chains in the NTD at $\mathrm{pH} 6$ is still incomplete and may eventually come from highresolution spectroscopic techniques such as $\mathrm{NMR}^{17,20}$.

Other ionizable side chains also contribute to assembly. We find that mutation of R60 and K65 results in substantial destabilization of the dimer by free energy increments of $\sim 2 \mathrm{kcal} \mathrm{mol}^{-1}$ (Fig. 5b). Positively charged R60 and K65 form intersubunit salt bridges with D39 and D40 (Fig. 6b). The stabilizing effect of these salt bridges has recently been addressed by computer simulations ${ }^{21}$. We also found that mutation of K93 destabilizes the dimer (Fig. 5b). However, this effect is difficult to interpret because the $\mathrm{K} 93$ side chain is in a buried position (Fig. 6c) and its mutation to alanine may possibly induce a structural perturbation. H6A and E79Q have values of $k_{\mathrm{a}}$ and $k_{\mathrm{d}}$ that are not substantially different from those of the pseudo wildtype protein. This corroborates findings from previous experimental studies where both mutants were still able to form dimers ${ }^{7,18}$.

The second question surrounding the mechanism of NTD assembly involves the actual association process itself that follows the protonation event(s). The universal theory of protein-protein association is well established. The shape of most proteins can be approximated as globules and their rates of random collision in aqueous solution is estimated from the Einstein-Smoluchowski equation to $\sim 6 \times 10^{9} \mathrm{M}^{-1} \mathrm{~s}^{-1}$ at $25^{\circ} \mathrm{C}$ (ref. 22). Protein association, however, rarely reaches this speed limit set by diffusion. Protein association has strict geometric constraints where complementary shapes require well-defined orientations before they can assemble. The probability of correct orientation of interfaces during a random collision is estimated to $\sim 1.5 \times 10^{-5}$ and results in the basal rate constant of protein-protein association of $\sim 10^{5} \mathrm{M}^{-1} \mathrm{~s}^{-1}$ (refs 15,22,23). Here we observe an unusually high NTD association rate constant of $3 \times 10^{8} \mathrm{M}^{-1} \mathrm{~s}^{-1}$, which is three orders of magnitude faster than the basal value and close to the diffusion speed limit. Favourable intermolecular Coulomb forces originating from opposite charges on protein interfaces can accelerate association to beyond the basal value ${ }^{15}$. This accelerating effect, however, is commonly screened by electrolytes in solution with numerous examples reported in the literature ${ }^{15,16,24-28}$. Surprisingly, we find that association of the NTD is insensitive to such screening (Fig. 4). But IS substantially accelerates NTD dissociation (Fig. 4). This behaviour is the exact opposite of what is commonly observed $^{15}$. Findings are corroborated by results from mutagenesis: deletion of NTD side chain charges have virtually no influence on the rate constant of association but accelerate dissociation (Fig. 5a,b). Previous structural studies analysed the distribution of side chain charges on the surface of the $\mathrm{NTD}^{7}$. The authors found that opposite charges cluster on opposing poles of the association interface, thereby generating a unique macromolecular dipole ${ }^{7}$. The anti-parallel symmetry of the interface leads to a favourable dipole-dipole interaction (Fig. 6c). This dipole interaction may provide the alignment force for accelerated association and explain its unusual insensitivity to dielectric screening. Early simulations on cytochrome $c$ peroxidase emphasized the importance of dipolar interactions as steering force in proteins ${ }^{29}$. Gilson et al..$^{30}$ predicted from calculations of protein electrostatics that for many interactions involving dipoles, solvent screening can essentially be ignored. In line with this argument, Lockhart and $\mathrm{Kim}^{31}$ found experimental evidence that, in contrast to charges, helix dipole interactions in proteins are hardly screened by IS.

Findings may have implications in the synthesis of silk fibres by web spiders. For spiders, the speed of dragline synthesis is vital. The fibre is being used both as web frame and the spider's lifeline. At a vertical fall and during roping, reeling speed can exceed $1 \mathrm{~m} \mathrm{~s}^{-1}$ (refs $1,32,33$ ). At such high reeling speed and rate of fibre synthesis, the residence time of spidroins in the acidified NTD assembly zone of the duct is only about $1 \mathrm{~ms}$ depending on spider size. Spidroin NTDs must therefore associate very quickly to assist silk synthesis. The spinning duct of web spiders contains up to $50 \%$ spidroin ${ }^{1}$. With a spidroin molecular weight of $\sim 300 \mathrm{kDa}$, the concentration equals $\sim 1 \mathrm{mM}$. Together with the basal rate constant of protein-protein association of $\sim 10^{5} \mathrm{M}^{-1} \mathrm{~s}^{-1}$ (refs 15,22,23), the concentration yields a basal association time of $\sim 10 \mathrm{~ms}$, which is already too slow for rapid fibre synthesis. The association of the NTD in context of an intact spidroin in the spinning gland may even occur significantly slower than estimated from the basal rate constant of protein association. Although the NTD is an autonomously folded, structurally independent domain it takes only $\sim 5 \%$ of a spidroin sequence. Its mobility could additionally be slowed by the presence of the extended repetitive domains that undergo structural transitions in the gland. Moreover, the high spidroin concentration leads to unusual viscid solution properties ${ }^{1}$ that will further slow diffusional motions of the NTDs. These considerations emphasize the requirement of accelerated NTD association beyond the basal value if it should assist the process of silk synthesis at high reeling speed. Acceleration by a common charge-driven process would be perturbed by the presence of physiological electrolytes in the spinning duct ${ }^{34}$. In the distal part of the duct where the silk fibre is formed, water is recovered from the spidroin solution ${ }^{1,4}$ leading to even an increase of IS. A macromolecular NTD dipole interaction that resists dielectric screening could accelerate association irrespective of IS, as observed in our experiments, and thus facilitate the synthesis of dragline silk at high reeling speed. Sequence conservation of NTDs from various silk glands and spider species suggests that such process could be a universal feature of silk fibre synthesis by web spiders.

\section{Methods}

Synthesis and mutagenesis and fluorescence modification of the NTD. The complementary DNA of the NTD from spidroin 1 of the major ampullate gland of the nursery web spider E. australis was purchased as synthetic gene, codon optimized for expression in Escherichia coli bacterial cells (GeneArt, Life Technologies). The NTD gene containing an N-terminal $\mathrm{His}_{6}$-tag followed by the thrombin recognition sequence (LVPRGS) for proteolytic cleavage was cloned into a modified pRSETA vector (Invitrogen). Single-point mutants were generated using the QuikChange mutagenesis protocol (Stratagene). NTD and mutants thereof were overexpressed in E. coli C41 (DE3) cells and isolated from clarified cell lysate by nickel nitrilotriacetic acid affinity chromatography, followed by proteolytic removal of the $\mathrm{His}_{6}$-tag using thrombin from bovine plasma (Sigma). The thrombindigested eluate from affinity chromatography was dialyzed into $20 \mathrm{mM} \mathrm{MES} \mathrm{pH}$ 6.0 , subsequently applied to an anion-exchange chromatography column (Poros HQ resin equilibrated with $20 \mathrm{mM}$ MES $\mathrm{pH}$ 6.0) and eluted with a gradient from 0 to $500 \mathrm{mM}$ sodium chloride. Pooled NTD fractions were purified to homogeneity using size exclusion chromatograpy (SEC) on a Superdex 75 column (GE Healthcare) equilibrated with $200 \mathrm{mM}$ ammonium bicarbonate. The pooled fractions from SEC were lyophilized and stored at $-20^{\circ} \mathrm{C}$. NTD-Q50C and mutants thereof were modified with the thiol-reactive maleimide derivative of the oxazine fluorophore AttoOxa11 (Atto-Tec). Labelling was carried out using a 10-fold molar excess of fluorophore reacted for $2.5 \mathrm{~h}$ at $25^{\circ} \mathrm{C}$ in $50 \mathrm{mM} \mathrm{3-(morpholino)propa-}$

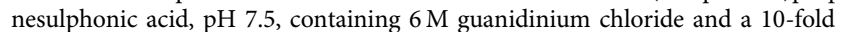
molar excess of tris(2-carboxyethyl)phosphine (Sigma) to prevent thiol oxidation. Labelled protein was isolated from unreacted dye using SEC.

Steady-state and time-resolved fluorescence experiments. Steady-state absorption and fluorescence emission spectra were recorded on a V-650 spectrophotometer and a FP-6500 spectrofluorimeter (Jasco). Association and dissociation kinetics were measured using stopped-flow fluorescence spectroscopy. Trp fluorescence data of NTD were recorded on an Applied Photophysics (SX 18MV) machine equipped with a xenon-lamp as excitation source. AttoOxal1 fluorescence data of $\mathrm{NTD}^{\mathrm{F}}$ were recorded on a SFM-2000 machine (BioLogic Instruments) using a diode at $639 \mathrm{~nm}$ as excitation source. Samples were filtered through $0.2 \mu \mathrm{m}$ syringe filters before measurement. For determination of $k_{\mathrm{a}}$ of NTD, NTD ${ }^{\mathrm{F}}$ and mutants thereof, protein samples were prepared at varying concentration in $10 \mathrm{mM}$ 
MES pH 7.0 with IS adjusted to $150 \mathrm{mM}$ and rapidly mixed into $50 \mathrm{mM}$ MES buffer at pH 6.0 using a volumetric mixing ratio of 1:10. For measurement of $k_{\mathrm{d}}$ of $\mathrm{NTD}^{\mathrm{F}}$ and mutants thereof, $100 \mathrm{nM}$ samples were prepared in $50 \mathrm{mM}$ MES pH 6.0 and chased with $5 \mu \mathrm{M}$ unlabelled NTD prepared in the same buffer, using a volumetric mixing ratio of 1:10. IS of buffers was adjusted using potassium chloride. All measurements were done at $25^{\circ} \mathrm{C}$.

Data analysis. Kinetic transients were fitted to exponential decay and rise functions. Values of $k_{\mathrm{a}}$ of $\mathrm{NTD}, \mathrm{NTD}^{\mathrm{F}}$ and mutants thereof were determined from the slopes of linear data fits to the concentration dependencies of $k_{\text {obs }}$ (five different concentrations each). Values of $k_{\mathrm{d}}$ from chasing experiments were determined as average values of observed rate constants from three measurements. The equilibrium dissociation constant, $K_{\mathrm{d}}$, and the free energy of dimerization, $\Delta G$, was calculated from $k_{\mathrm{a}}$ and $k_{\mathrm{d}}$ :

$$
\Delta G=-R T \ln \left(K_{d}\right)=-R T \ln \left(k_{d} / k_{a}\right) .
$$

$R$ is the gas constant and $T$ the temperature $(298 \mathrm{~K})$. The change of free energy on mutation, $\Delta \Delta G$, was calculated by:

$$
\Delta \Delta G=R T \ln \left(K_{d}^{M} / K_{d}^{W T}\right)
$$

where $K_{\mathrm{d}}^{\mathrm{WT}}$ and $K_{\mathrm{d}}^{\mathrm{M}}$ are the $K_{\mathrm{d}}$ values of $\mathrm{NTD}^{\mathrm{F}}$ (WT) and a single-point mutant $(\mathrm{M})$

\section{References}

1. Vollrath, F. \& Knight, D. P. Liquid crystalline spinning of spider silk. Nature 410, 541-548 (2001).

2. Gosline, J. M., Guerette, P. A., Ortlepp, C. S. \& Savage, K. N. The mechanical design of spider silks: from fibroin sequence to mechanical function. J. Exp. Biol. 202, 3295-3303 (1999).

3. Hinman, M. B., Jones, J. A. \& Lewis, R. V. Synthetic spider silk: a modular fiber. Trends Biotechnol. 18, 374-379 (2000).

4. Heim, M., Keerl, D. \& Scheibel, T. Spider silk: from soluble protein to extraordinary fiber. Angew. Chem. Int. Ed. Engl. 48, 3584-3596 (2009).

5. Eisoldt, L., Thamm, C. \& Scheibel, T. The role of terminal domains during storage and assembly of spider silk proteins. Biopolymers 97, 355-361 (2011).

6. Hagn, F. et al. A conserved spider silk domain acts as a molecular switch that controls fibre assembly. Nature 465, 239-242 (2010).

7. Askarieh, G. et al. Self-assembly of spider silk proteins is controlled by a pHsensitive relay. Nature 465, 236-238 (2010).

8. Vollrath, F., Knight, D. P. \& Hu, X. W. Silk production in a spider involves acid bath treatment. Proc. R. Soc. Lond. B 265, 817-820 (1998).

9. Hagn, F., Thamm, C., Scheibel, T. \& Kessler, H. pH-dependent dimerization and salt-dependent stabilization of the $\mathrm{N}$-terminal domain of spider dragline silk--implications for fiber formation. Angew. Chem. Int. Ed. Engl. 50, 310-313 (2011)

10. Gaines, W. A., Sehorn, M. G. \& Marcotte, Jr. W. R. Spidroin N-terminal domain promotes a $\mathrm{pH}$-dependent association of silk proteins during selfassembly. J. Biol. Chem. 285, 40745-40753 (2010).

11. Packard, B. Z., Toptygin, D. D., Komoriya, A. \& Brand, L. Profluorescent protease substrates: intramolecular dimers described by the exciton model. Proc. Natl Acad. Sci. USA 93, 11640-11645 (1996).

12. Bollmann, S., Löllmann, M., Sauer, M. \& Doose, S. Dimer formation of organic fluorophores reports on biomolecular dynamics under denaturing conditions. Phys. Chem. Chem. Phys. 13, 12874-12882 (2011).

13. Jaudzems, K. et al. pH-dependent dimerization of spider silk N-terminal domain requires relocation of a wedged tryptophan side chain. J. Mol. Biol. 422, 477-487 (2012)

14. Debye, P. \& Hückel, E. Zur Theorie der Elektrolyte. Gefrierpunktserniedrigung und verwandte Erscheinungen. Physik. Z 24, 185-206 (1923).

15. Schreiber, G., Haran, G. \& Zhou, H. X. Fundamental aspects of protein-protein association kinetics. Chem. Rev. 109, 839-860 (2009).

16. Gianni, S. et al. The kinetics of PDZ domain-ligand interactions and implications for the binding mechanism. J. Biol. Chem. 280, 34805-34812 (2005).

17. Pace, C. N., Grimsley, G. R. \& Scholtz, J. M. Protein ionizable groups: $\mathrm{pK}$ values and their contribution to protein stability and solubility. J. Biol. Chem. 284, 13285-13289 (2009).
18. Landreh, M. et al. A pH-dependent dimer lock in spider silk protein. J. Mol. Biol. 404, 328-336 (2010).

19. Wallace, J. A. \& Shen, J. K. Unraveling a trap-and-trigger mechanism in the pH-sensitive self-assembly of spider silk proteins. J. Phys. Chem. Lett. 3, 658-662 (2012).

20. Arbely, E., Rutherford, T. J., Sharpe, T. D., Ferguson, N. \& Fersht, A. R. Downhill versus barrier-limited folding of BBL 1 : energetic and structural perturbation effects upon protonation of a histidine of unusually low pKa. J. Mol. Biol. 387, 986-992 (2009).

21. Gronau, G., Qin, Z. \& Buehler, M. J. Effect of sodium chloride on the structure and stability of spider silk's N-terminal protein domain. Biomater. Sci. 1, 276-284 (2013).

22. Janin, J. The kinetics of protein-protein recognition. Proteins 28, 153-161 (1997).

23. Schlosshauer, M. \& Baker, D. Realistic protein-protein association rates from a simple diffusional model neglecting long-range interactions, free energy barriers, and landscape ruggedness. Protein Sci. 13, 1660-1669 (2004).

24. Alsallaq, R. \& Zhou, H. X. Prediction of protein-protein association rates from a transition-state theory. Structure 15, 215-224 (2007).

25. Schreiber, G. \& Fersht, A. R. Rapid, electrostatically assisted association of proteins. Nat. Struct. Biol. 3, 427-431 (1996).

26. Selzer, T., Albeck, S. \& Schreiber, G. Rational design of faster associating and tighter binding protein complexes. Nat. Struct. Biol. 7, 537-541 (2000).

27. Sheinerman, F. B., Norel, R. \& Honig, B. Electrostatic aspects of protein-protein interactions. Curr. Opin. Struct. Biol. 10, 153-159 (2000).

28. Rogers, J. M., Steward, A. \& Clarke, J. Folding and binding of an intrinsically disordered protein: fast, but not 'diffusion-limited'. J. Am. Chem. Soc. 135, 1415-1422 (2013).

29. Northrup, S. H., Reynolds, J. C. L., Miller, C. M., Forrest, K. J. \& Boles, J. O. Diffusion-controlled association rate of cytochrome-C and cytochrome-C peroxidase in a simple electrostatic model. J. Am. Chem. Soc. 108, 8162-8170 (1986).

30. Gilson, M. K., Rashin, A., Fine, R. \& Honig, B. On the calculation of electrostatic interactions in proteins. J. Mol. Biol. 184, 503-516 (1985).

31. Lockhart, D. J. \& Kim, P. S. Electrostatic screening of charge and dipole interactions with the helix backbone. Science 260, 198-202 (1993).

32. Breslauer, D. N., Lee, L. P. \& Muller, S. J. Simulation of flow in the silk gland. Biomacromolecules 10, 49-57 (2009).

33. Ortlepp, C. S. \& Gosline, J. M. Consequences of forced silking. Biomacromolecules 5, 727-731 (2004).

34. Knight, D. P. \& Vollrath, F. Changes in element composition along the spinning duct in a Nephila spider. Naturwissenschaften 88, 179-182 (2001).

\section{Acknowledgements}

We thank the Deutsche Forschungsgemeinschaft (DFG) for financial support (Grant number NE 1201/3-1).

\section{Author contributions}

H.N. designed research; S.S., F.U.Z., C.M.J. and H.N. performed research; S.S., C.M.J. and H.N. analysed data; H.N. wrote the paper.

\section{Additional information}

Supplementary Information accompanies this paper at http://www.nature.com/ naturecommunications

Competing financial interests: The authors declare no competing financial interests

Reprints and permission information is available online at http://npg.nature.com/ reprintsandpermissions/

How to cite this article: Schwarze, S. et al. The N-terminal domains of spider silk proteins assemble ultrafast and protected from charge screening. Nat. Commun. 4:2815 doi: $10.1038 /$ ncomms3815 (2013). 